'Residente de Neurología, Facultad de Medicina, Universidad de Los Andes. Santiago, Chile.

${ }^{2}$ Clínica Alemana de Santiago, Facultad de Medicina Clínica Alemana, Universidad del Desarrollo, Santiago, Chile. Liga Chilena contra la Epilepsia de Santiago. Santiago, Chile. ${ }^{3}$ Liga Chilena contra la Epilepsia, Clínica Universidad de los Andes,

Clínica Dávila. Santiago, Chile.

No hay fuente de apoyo financiero.

Recibido el 9 de julio de 2017, aceptado el 7 de junio de 2018 .

Correspondencia a:

Dr. Mauricio Velásquez Cabrera

Dirección Facultad de Medicina Universidad de Los Andes Monseñor Alvaro del Portillo 12455, Las Condes, Santiago, Chile. mfvelasquezc@gmail.com

\section{Muerte súbita en epilepsia}

\author{
MAURICIO VELÁSQUEZ¹, ALEJANDRO DE MARINIS², \\ EVELYN BENAVIDES ${ }^{3}$
}

\section{Sudden death in epilepsy}

The risk of sudden unexpected death in patients with epilepsy (SUDEP), is 20 to 25 times greater than in the general population. This increased risk is seen specially in refractory epilepsy, with an incidence of 9:1,000 patients-years. Risk factors have been established based on retrospective studies, finding that the frequency of seizures, specially generalized tonic clonic seizures, is the most important one. The physiopathological mechanism of SUDEP is not yet fully understood. Autonomic system abnormalities, as well as cardiac and respiratory changes have been found. The finding of new molecular biomarkers to identify patients with increased risk should be a priority. Treatment is based in the management of risk factors, although clear recommendations are hard to establish given the low level of evidence.

(Rev Med Chile 2018; 146: 902-908)

Key words: Death, sudden; Epilepsy; Seizures.
4 1 concepto de muerte súbita en epilepsia, SUDEP por sus siglas en inglés (Sudden Unexpected Death in Epilepsy), se define como la muerte que se produce de forma súbita, inesperada, no traumática ni por asfixia en pacientes con epilepsia, sin considerar como relevante la presencia de testigos, o la evidencia de crisis epiléptica previa, pero se excluyen las muertes secundarias a estatus epiléptico ${ }^{1}$. En estos pacientes el estudio anatomopatológico post mortem no debe revelar signos de una causa de muerte toxicológica o anatómica subyacente.

A pesar de ser un concepto relativamente nuevo, podemos encontrar múltiples referencias en la historia de la medicina. Se le atribuye la primera descripción de muerte súbita a Hipócrates en el siglo IV AC, quien estableció que "aquellos que son sujetos de frecuentes y severos ataques de desvanecimiento sin causa obvia, mueren súbitamente". $\mathrm{Su}$ relación con la epilepsia fue reconocida en el año 1854, cuando el psiquiatra francés Louis Delasiauve realiza la primera descripción formal de SUDEP, planteando que la muerte en estos pacientes podría ser secundaria a sofocación ${ }^{2}$. En el año 1902, William Spratling describió casos de muerte súbita en pacientes con epilepsia institu- cionalizados, estableciendo ésta como causa de muerte en 3 a $4 \%$ de los que sufren esta enferme$\mathrm{dad}^{3}$. Por un tiempo este concepto es dejado de lado, recobrando importancia entre 1970 y 1980, observándose un aumento en la investigación a nivel mundial ${ }^{1}$.

\section{Epidemiología}

La mortalidad global en los pacientes con epilepsia es 2 a 3 veces mayor que en la población general $^{4,5}$, mientras que el riesgo de muerte súbita esta aumentado 20 a 25 veces $^{6,7}$.

Entre las causas de muerte más importantes se encuentra SUDEP con $2-18 \%$ de los casos, otras causas como estatus epiléptico, accidentes y suicidio, han demostrado tener una prevalencia menor en estudios de países desarrollados ${ }^{8,9}$. Se debe considerar que probablemente estas cifras estén subestimadas, por lo que una mejor monitorización y vigilancia activa por parte del personal de salud, permitiría obtener datos más confiables.

La incidencia de SUDEP en diferentes estudios varía entre 1:500 a 1:1.000 pacientes al año. Los menores índices se observan en estudios poblacio- 
Tabla 1. Categorías Diagnósticas de SUDEP

\begin{tabular}{|ll|}
\hline Categorías diagnósticas & Criterios \\
SUDEP Definitivo & $\begin{array}{l}\text { Cumple criterios clínicos y cuenta con estudio anatomo patológico negativo para otra } \\
\text { causa de muerte }\end{array}$ \\
\hline SUDEP Probable & Cumple criterios clínicos pero no se cuenta con estudio anatomo patológico \\
\hline SUDEP Posible & $\begin{array}{l}\text { Falta información para determinar con certeza SUDEP, o existe otra causa probable de } \\
\text { muerte (aunque no confirmada) }\end{array}$ \\
\hline
\end{tabular}

nales, mientras que los mayores los encontramos en pacientes con epilepsia refractaria y candidatos a cirugía de epilepsia, llegando a ser de 9:1.000 pacientes-año ${ }^{6,7,10,11}$. Afecta principalmente a pacientes jóvenes, en especial entre los 20 y 40 años, sin una predilección según género ${ }^{12}$.

El impacto principal que tiene SUDEP está dado por los años de vida potencialmente perdidos (AVPP). Es una de las principales causas de muerte en pacientes con epilepsia, y ocurre preferentemente en personas jóvenes. En Estados Unidos de Norteamérica se ha estimado que se ubica en el segundo lugar de AVPP dentro de las patologías neurológicas con alrededor de 100.000 AVPP, solo por detrás del accidente cerebrovascular que llega a los 189.000 y siendo seguido por la esclerosis lateral amiotrófica con $57.000^{12}$.

\section{Diagnóstico}

En el diagnóstico de SUDEP se han establecido tres categorías diagnósticas (Tabla 1): SUDEP definitivo, si cumple con todos los criterios clínicos (Tabla 2) y cuenta con estudio anatomopatológico; Probable, si cumple con todos los criterios clínicos pero no se realizó estudio post mortem; y Posible, cuando falta información suficiente para establecer el diagnóstico, o existe otra causa probable de muerte ${ }^{13,14}$.

El estudio anatomopatológico debe incluir un análisis macroscópico e histológico. Para esto es muy importante enviar la información necesaria al patólogo previo a la autopsia, incluyendo tipo de crisis, su duración y frecuencia, fármacos antiepilépticos (FAE) que utilizaba el paciente, la causa de su epilepsia (cuando se conoce), además de las circunstancias de la muerte y condiciones médicas relevantes (cardiopatía, consumo de alcohol, etc.).

La evaluación post mortem negativa no traduce

Tabla 2. Criterios clínicos de SUDEP

Criterios clínicos de SUDEP

- Diagnóstico de epilepsia establecido

- Muerte inesperada estando en condición de salud razonable

- Muerte súbita, habitualmente en minutos

- Muerte ocurre durante actividades normales, en circunstancias benignas

- No se encuentra causa estructural de muerte

- Muerte no se debe a status epiléptico o trauma secundario a la crisis

necesariamente la indemnidad del cerebro. Diferentes estudios han reportado alteraciones en el sistema nervioso central hasta en $70 \%$ de los pacientes, siendo las lesiones más frecuentemente encontradas atrofia hipocampal o cortical, estigmas de trauma antiguo, malformaciones corticales, hemangiomas y tumores ${ }^{15,16}$. A su vez, es frecuente observar alteraciones cardíacas como vacuolización de miocitos, fibrosis del tejido miocárdico y la presencia de infiltrados inflamatorios ${ }^{15}$.

\section{Factores de riesgo}

Diferentes factores han sido postulados, los que pueden ser divididos en aquellos relacionados con las crisis, el tratamiento de la epilepsia, y características específicas de cada paciente.

La evidencia que avala estos factores está constituida principalmente por trabajos retrospectivos, los que tienen sesgos propios de este tipo de estudios ${ }^{7,17-20}$. Analizando todos ellos podemos categorizar dentro de cada grupo algunos factores de alto impacto y otros de menor relevancia 
Tabla 3. Factores de riesgo SUDEP

\begin{tabular}{|lll|}
\hline Categorías & Alto impacto & Bajo impacto \\
\hline Crisis epilépticas & Crisis TCG & Crisis nocturnas \\
& Mayor frecuencia de crisis & \\
Tratamiento de epilepsia & Refractariedad & Inicio de epilepsia $<16$ años \\
& Mala adherencia a tratamiento (pseudorefractariedad) & Duración de epilepsia $>15$ años \\
& Bajos niveles plasmáticos de FAE & \\
\hline Características de pacientes & Edad entre 20 y 40 años & Asma \\
& Abuso de alcohol & Demencia \\
\hline
\end{tabular}

TCG: tónico clónicas generalizadas; FAE: fármacos antiepilépticos.

(Tabla 3), basados principalmente en la calidad de los estudios que los reportan, ya que valores estadísticos reales han sido pobremente establecidos.

En relación a las crisis, el factor más importantes es su mal control, lo que se traduce en una mayor frecuencia de eventos epilépticos, ya sea por refractariedad de la enfermedad o por mala adherencia a tratamiento (pseudorefractariedad). El tipo de crisis y la hora del día en que se presentan también han sido relacionados, siendo las tónico clónicas generalizadas las de mayor impacto, mientras que las crisis nocturnas en menor medida también se han asociado con mayor frecuencia de SUDEP.

Considerando la epilepsia y su tratamiento, como se mencionó, la refractariedad y pseudorefractariedad han sido establecidas como factores de riesgo importantes, así como también mantener bajos niveles plasmáticos de FAE.

El inicio de la enfermedad antes de los 16 años, y su duración por más de 15 años en menor grado también se relacionan.

Otros estudios han asociado la politerapia, y cambios frecuentes de FAE con SUDEP, aunque se ha demostrado que estos factores se relacionan a un mal control de crisis, por lo que ya no son considerados como factores de riesgo independientes $^{19}$. Algunos fármacos específicos fueron considerados como factores de riesgo, específicamente lamotrigina y carbamazepina, debido a que pueden presentar como efectos adversos a nivel cardiovascular, la prolongación del QT, arritmias y trastornos de la conducción. Sin embargo, los estudios no han encontrado una diferencia significativa en las tasas de SUDEP en los pacientes tratados con estos medicamentos ${ }^{19,20}$.

De las características propias de los pacientes, la edad entre 20 y 40 años ha sido asociada a SUDEP, además de patologías concomitantes, siendo el abuso de alcohol el de mayor impacto, otros como demencia y asma también se han reportado.

Un estudio del año 2016 no encontró una asociación de otros factores de riesgo anteriormente planteados, como retardo mental, género femenino, y uso concomitante de antidepresivos, con una mayor incidencia de SUDEP ${ }^{17}$.

\section{Fisiopatología}

Hasta la fecha aún no se han dilucidado completamente los mecanismos involucrados en SUDEP, por qué afecta a algunos pacientes y que relación existe con los factores de riesgo identificados.

Conocer la fisiopatología de SUDEP, permitiría determinar nuevos factores de riesgo posibles de tratar e identificar nuevos objetivos en la terapia, por lo que han surgido diversas teorías y múltiples estudios.

Se han logrado identificar alteraciones principalmente en 3 mecanismos; cardiovasculares (taqui y bradiarritmias, asistolia ictal durante la crisis), respiratorios (apnea central, edema pulmonar neurogénico), y en el sistema autónomo (predominio simpático y disfunción vagal sostenida). Se cree que todas ellas probablemente serían gatilladas por las crisis convulsivas.

Uno de los principales estudios en SUDEP es el MORTEMUS ${ }^{21}$, trabajo retrospectivo multi- 
céntrico que evaluó a pacientes que sufrieron de SUDEP mientras estaban siendo monitorizados en unidades de epilepsia. Logró reunir 29 casos, siendo la mayor serie publicada; sin embargo, se debe considerar que presenta algunos sesgos importantes, como la falta de estudio anatomopatológico en la mitad de los casos presentados, y la falta de datos sobre presión arterial y presión de perfusión cerebral entre otros. En la mayoría de los pacientes se observó una progresión posterior de crisis, taquipnea, disfunción cardíaca, luego apnea y finalmente paro cardiorrespiratorio. En algunos casos el evento inicial fue una apnea central post ictal. También se observó la presencia de bradiarritmia $\mathrm{o}$ asistolia ictal. En ningún paciente se logró determinar patrones electroencefalográficos predictores de SUDEP. Los registros mostraron una ventana de tiempo acotada para poder realizar maniobras de resucitación a los pacientes, que en general no supera los 11 min desde el evento inicial.

En relación a la disfunción cardiovascular, no se ha logrado identificar alteraciones preexistentes relacionadas con SUDEP, por lo que el foco de los estudios ha estado en determinar los cambios secundarios a las crisis epilépticas reiteradas ${ }^{22}$. Entre ellas se han descrito arritmias por alteraciones en canales de sodio y potasio, observándose una sobre expresión de isoformas reguladas por el gen SCN5A y KCNQ1 respectivamente, lo que traería como consecuencia un QT prolongado. Además se ha observado la expresión en tejido cardíaco de canales de sodio neuronales dependiente del gen SCN1A, lo que aumenta el riesgo de arritmias. Estos genes han sido postulados como potenciales biomarcadores de riesgo de SUDEP, sin embargo, su rol concreto en los pacientes no ha podido ser establecido $^{22,23}$.

El remodelamiento cardíaco ocurre en conjunto con la evolución de la epilepsia y se acentuaría a mayor cantidad de crisis. También se observan alteraciones cardiovasculares secundarias a la disfunción autonómica, como por ejemplo una disminución en la variabilidad de la frecuencia cardíaca.

En lo respiratorio, destaca la apnea o hipoapnea central, secundaria a la supresión de los centros respiratorios en el tronco del encéfalo por la depolarización propagada debido a las crisis, lo que se ha observado en modelos animales ${ }^{24}$. Además se produciría una alteración en el control serotoninérgico de la respiración (quimiorreceptores de $\mathrm{CO}_{2}$ y pH). En modelos animales, se suprimió la expresión de receptores de serotonina, produciendo hipoventilación, apnea y disminución de respuesta a hipercapnia, observándose también mayor número de crisis y una mayor susceptibilidad a muerte prematura ${ }^{25}$. Otro mecanismo de SUDEP postulado es la apnea obstructiva, dada la relación de la postura en prono observada en los pacientes que sufren de esta enfermedad. Por último existe también un daño pulmonar primario por la alteración en la irrigación y drenaje venoso de estos órganos, y la presencia de edema pulmonar neurogénico, aunque la evidencia en autopsias indica que este edema es leve y difícilmente sería la causa de muerte súbita.

Otros mecanismos planteados incluyen la liberación exagerada de opioides endógenos y adenosina, involucrados en el cese de las crisis y responsables de la supresión post ictal del electroencefalograma, ambos mecanismos se encuentran aun en etapa de estudio.

\section{Tratamiento}

El tratamiento de SUDEP se centra en la prevención, aunque debe considerarse que hasta la fecha no existe evidencia que avale alguna intervención específica.

De todas formas el énfasis actual se pone en el manejo de los factores de riesgo conocidos, en especial el control de crisis, y en el manejo de vías descritas como posibles mecanismos fisiopato$\operatorname{lógicos}^{26}$.

En 2016 fue publicada una revisión Cochrane que evaluó tratamientos para la prevención de SUDEP. Se consideró: cirugía de la epilepsia, sensores de crisis, supervisión nocturna, almohadas de seguridad, inhibidores de recaptación de serotonina, inhibidores de adenosina y de opiáceos ${ }^{27}$. El único de estos factores que presentó evidencia a su favor fue la supervisión nocturna de los pacientes, que incluye el chequeo regular durante la noche, evitar la posición de prono y conocer maniobras de reanimación. Los inhibidores de la recaptación de serotonina mostraron un efecto importante en la reducción de la severidad de la hipoxemia ictal asociada con las crisis, sin embargo, este efecto ha podido ser demostrado solo en pacientes con crisis focales, por lo que su real utilidad en SUDEP es aún incierta ${ }^{28}$. 
En pacientes con epilepsia refractaria se debe revisar la terapia farmacológica de forma activa, implementando cambios para buscar un mejor control de crisis. Un metaanálisis publicado en 2011 mostró que este enfoque puede llegar a reducir hasta 7 veces la incidencia de SUDEP $^{29}$. Además de la optimización del tratamiento es importante la adherencia de los pacientes a éste y el control médico regular, factores que pueden mejorarse con una buena educación al paciente y su familia. Por otra parte, es relevante reforzar la importancia de los hábitos saludables, en especial evitar el consumo de alcohol y una buena higiene de sueño.

Otra medida para disminuir las crisis utilizada sólo en pacientes con epilepsia refractaria es la cirugía de la epilepsia. Se evaluó en un estudio la mortalidad de pacientes con epilepsia refractaria que fueron derivados a cirugía (resectiva y callosotomía) y el control de crisis logrado en el post operatorio. Se encontró una disminución significativa de la mortalidad (incluyendo SUDEP) en pacientes con cirugía resectiva que no tuvieron recurrencia de crisis, obteniendo un valor de 0,8 a 1 por 1.000 pacientes-año, mientras que al presentar recurrencias la mortalidad alcanzó un 9,3 por 1.000 pacientes-año. En cuanto a la callosotomía, se observó una mortalidad de 4,5 por 1.000 pacientes-año para aquellos que no presentaron recurrencias, mientras que en aquellos que si recurrieron, la mortalidad fue de 22 por 1.000 pacientes-año ${ }^{30}$. La no recurrencia de crisis post cirugía es el factor determinante en la disminución de la mortalidad, sin embargo, esto no se logra en todos los casos, por lo que seleccionar adecuadamente los pacientes a intervenir quirúrgicamente es fundamental.

También se ha evaluado el impacto de la estimulación del nervio vago, sin embargo, los estudios a la fecha no han encontrado una diferencia significativa en la reducción de las tasas de SUDEP $^{31}$.

Se han reportado algunas terapias invasivas, como la implantación de marcapasos para el manejo de asistolía ictal, y el uso de neuroestimuladores implantables. También se han probado monitores multimodales que incluyen análisis de función motora con acelerómetros, de apnea, y de electrocardiograma, los que podrían facilitar la rápida intervención, a pesar de que han presentado dificultades para detectar eventos correspondientes a falsos positivos. A la fecha, el nivel de evidencia es bajo, y su impacto aún no ha sido establecido, por lo que su uso debiera ser recomendado caso a caso y no como norma en el manejo de esta patología.

Otro punto relevante en el manejo de SUDEP, son las intervenciones comunitarias. Su efectividad no ha sido probada, sin embargo, campañas publicitarias enfocadas en mejorar los hábitos de los pacientes, su adherencia a tratamiento y la supervisión en caso de epilepsias refractarias podrían ser de utilidad. Además, el avanzar hacia el acceso universal al tratamiento es una medida poblacional importante para reducir la mortalidad asociada a la epilepsia, incluyendo SUDEP ${ }^{26}$.

Hablar de SUDEP con los pacientes y sus familias es un tema difícil. Un estudio publicado en 2014 mostró que en EE.UU. y Canadá solo $6,8 \%$ de los neurólogos discuten SUDEP con casi todos sus pacientes, mientras que $11,6 \%$ nunca lo hace $^{32}$. La recomendación actual insta a los médicos a informar a todos los pacientes. Dentro de las principales razones se encuentra el entregar datos confiables, evitar información errónea y mejorar la adherencia a tratamiento. Sin embargo, algunos neurólogos lo evitan argumentando que aumenta la ansiedad y el estrés relacionado a la enfermedad y que sólo debiera ser discutido con pacientes seleccionados ${ }^{11}$. La manera de abordar el tema debe individualizarse caso a caso, sin existir formas estandarizadas para hacerlo.

\section{Conclusiones}

La muerte súbita en epilepsia es una patología relevante, con una incidencia probablemente subestimada dada la falta de registros confiables, que afecta principalmente al subgrupo de pacientes con epilepsia refractaria. Representa la causa más común de muerte en epilepsia de causa no traumática, y su impacto en AVPP es muy importante.

El principal factor de riesgo de SUDEP es la presencia de crisis tónico clónicas generalizadas frecuentes, por lo que el tratamiento se ha enfocado en su control.

Se está avanzando cada vez más en el conocimiento de su fisiopatología, sin embargo, aún queda un largo camino para dilucidar todos los mecanismos involucrados. Las políticas públicas relacionadas con el buen control de crisis y acceso 
a tratamiento efectivos probablemente tengan una incidencia en disminuir el riesgo de SUDEP, lo cual debería ser considerado en las intervenciones comunitarias en epilepsia.

\section{Referencias}

1. Nashef L. Sudden unexpected death in epilepsy: terminology and definitions. Epilepsia 1997; 38 (suppl 11): S6-S8.

2. Delasiauve LJF. Traité de l'épilepsie: Histoire, Traitement, Médecine Légale. Paris: Masson; 1984.

3. Spratling WP. The causes and manner of death in epilepsy. Med News 1902; 80: 1225-7.

4. Cockerell OC, Hart YM, Sander JW, Goodridge DM, Shorvon SD, Johnson AL. Mortality from epilepsy: results from a prospective population-based study. Lancet 1994; 344: 918-21.

5. Sillanpaa M, Shinnar S. Long-term mortality in childhood-onset epilepsy. N Engl J Med 2010; 363: 2522-9.

6. Thurman D, Logroscino G, Beghi E, Hauser W, Hesdorffer D, Newton C, et al. The burden of premature mortality of epilepsy in high-income countries: A systematic review from the Mortality Task Force of the International League Against Epilepsy. Epilepsy 2016. doi: 10.1111/epi.13604. [Epub ahead of print].

7. Tomson T, Walczak T, Sillanpaa M, Sander JW. Sudden unexpected death in epilepsy: a review of incidence and risk factors. Epilepsia 2005; 46 (suppl 11): 54-61.

8. Mohanraj R, Norrie J, Stephen LJ, Kelly K, Hitiris N, Brodie MJ. Mortality in adults with newly diagnosed and chronic epilepsy: a retrospective comparative study. Lancet Neurol 2006; 5: 481-7.

9. Hitiris N, Mohanraj R, Norrie J, Brodie MJ. Mortality in epilepsy. Epilepsy Behav 2007; 10 (3): 363-76.

10. Shorvon S, Tomson T. Sudden unexpected death in epilepsy. Lancet 2011; 378: 2028-38.

11. Marin V, Tatum W. Sudden Unexpected Death in Epilepsy (SUDEP) Are All Your Patients Informed? The Neurologist 2016; 21: 66-71.

12. Thurman DJ, Hesdorffer DC, French JA. Sudden unexpected death in epilepsy: assessing the public health burden. Epilepsia 2014; 55 (10): 1479-85.

13. Annengers J, United States Perspective on Definitions and Classifications. Epilepsia 1997; 38 (Suppl. 11): S9-S12.

14. Nashef L, So E, Ryvlin P, Tomson T, Unifying the definitions of sudden unexpected death in epilepsy. Epilepsia 2012; 53 (2): 227-33.

15. Thom M. The autopsy in sudden unexpected adult death: Epilepsy. Current Diagnostic Pathology 2007; 13 (5): 389-400.
16. Shields LB, Hunsaker DM, Hunsaker JC 3rd, Parker JC Jr. Sudden unexpected death in epilepsy: neuropathologic findings. Am J Forensic Med Pathol 2002; 23 (4): 307-14.

17. Shankar R, Walker M, McLean B, Laugharne R, Ferrand F, Hanna J, et al. Steps to prevent SUDEP: the validity of risk factors in the SUDEP and seizure safety checklist: a case control study. J Neurol 2016; 263 (9): 1840-6.

18. Hitiris N, Suratman S, Kelly K, Stephen LJ, Sills GJ, Brodie MJ. Sudden unexpected death in epilepsy: a search for risk factors. Epilepsy Behav 2007; 10: 138-41.

19. Hesdorffer DC, Tomson T. Sudden Unexpected Death in Epilepsy: Potential Role of Antiepileptic Drugs, CNS Drugs 2013; 27 (2): 113-9.

20. Tomson T, Hirsch LJ, Friedman D, Bester N, Hammer A, Irizarry M, et al. Sudden unexpected death in epilepsy in lamotrigine randomized-controlled trials. Epilepsia 2013; 54 (1): 135-40.

21. Ryvlin P, Nashef L, Lhatoo S, Bateman L, Bird J, Bleasel A, et al. Incidence and mechanisms of cardiorespiratory arrests in epilepsy monitoring units (MORTEMUS): a retrospective study. Lancet Neurol 2013; 12: 966-77.

22. Goldman A, Behr E, Semsarian C, Bagnall R, Sisodiya $\mathrm{S}$, Cooper P. Sudden unexpected death in epilepsy genetics: Molecular diagnostics and prevention. Epilepsia 2016; 57 (Suppl. 1): 17-25.

23. Dlouhy BJ, Gehlbach B, Richerson G. Sudden unexpected death in epilepsy: basic mechanisms and clinical implications for prevention. J Neurol Neurosurg Psychiatry 2015; 0: 1-12.

24. Aiba I, Noebels J. Spreading depolarization in the brainstem mediates sudden cardiorespiratory arrest in mouse SUDEP models. Sci Transl Med 2015; 7 (282): 282ra46.

25. Tupal S, Faingold C. Evidence supporting a role of serotonin in modulation of sudden death induced by seizures in DBA/2 mice. Epilepsia 2006; 47 (1): 21-6.

26. Tomson T, Surges R, Delamont R, Haywood S, Hesdorffer DC. Who to target in sudden unexpected death in epilepsy prevention and how? Risk factors, biomarkers, and intervention study designs, Epilepsia 2016; 57 (Suppl. 1): 4-16.

27. Maguire MJ, Jackson CF, Marson AG, Nolan SJ. Treatments for the prevention of Sudden Unexpected Death in Epilepsy (SUDEP). Cochrane Database Syst Rev 2016; 7: CD011792.

28. Bateman L, Li CS, Lin TC, Seyal M. Serotonin reuptake inhibitors are associated with reduced severity of ictal hypoxemia in medically refractory partial epilepsy. Epilepsia 2010; 51 (10): 2211-4.

29. Ryvlin P, Cucherat M, Rheims S. Risk of sudden unex- 
pected death in epilepsy in patients given adjunctive antiepileptic treatment for refractory seizures: a meta-analysis of placebo-controlled randomised trials. Lancet Neurol 2011; 10 (11): 961-8.

30. Sperling MR, Feldman H, Kinman J, Liporace JD, O'Connor MJ. Seizure control and mortality in epilepsy. Ann Neurol 1999; 46 (1): 45-50.
31. Granbichler C, Nashef L, Selway R, Polkey C. Mortality and SUDEP in epilepsy patients treated with vagus nerve stimulation. Epilepsia 2015; 56 (2): 291-6.

32. Friedman D, Donner E, Stephens D, Wright C, Devinski O. Sudden Unexpected Death in Epilepsy: knowledge and experience among US and Canadian neurologist. Epilepsy Behav 2014; 3535: 13-8. 